\title{
Treadmill exercise improves spatial learning ability by enhancing brain-derived neurotrophic factor expression in the attention-deficit/hyperactivity disorder rats
}

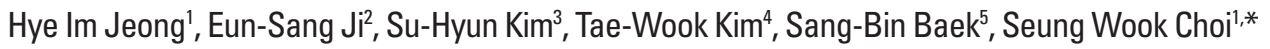 \\ 'Department of Physical Education, Graduate School, Sungshin Women's University, Seoul, Korea \\ 2Department of Physiology, College of Medicine, Kyung Hee University, Seoul, Korea \\ ${ }^{3}$ Department of Physical Education, Graduate School, Dankook University, Seoul, Korea \\ ${ }^{4}$ Department of Physical Education, College of Physical Education, Hanyang University, Seoul, Korea \\ ${ }^{5}$ Department of Psychiatry, Gangneung Asan Hospital, Ulsan University, Gangneung, Korea
}

Attention-deficit/hyperactivity disorder (ADHD) patients show learning difficulty and impulsiveness. Exercise is known to improve learning ability and memory function. In the present study, we investigated the duration-dependence of the effect of treadmill exercise on spatial learning ability in relation with brain-derived neurotrophic factor (BDNF) expression in ADHD rats. For this study, radial 8-arm maze test and western blot for BDNF and tyrosine kinase B (TrkB) were performed. Spontaneous hypertensive rats were used as the ADHD rats and Wistar-Kyoto rats were used as the control rats. The rats in the exercise groups were forced to run on a treadmill for $10 \mathrm{~min}, 30 \mathrm{~min}$, and $60 \mathrm{~min}$ once a day for 28 consecutive days. ADHD rats displayed impairment of spatial learning ability, in contrast treadmill exercise ameliorated impairment of spatial learning ability. Treadmill exercise for 30 min per day showed most potent ameliorating effect on impairment of spatial learning ability. BDNF and TrkB expressions in the hippocampus were decreased in the ADHD rats, in contrast treadmill exercise enhanced BDNF and TrkB expressions. Treadmill exercise for $30 \mathrm{~min}$ and for $60 \mathrm{~min}$ per day showed enhancing effects on BDNF and TrkB expressions. Treadmill exercise alleviated deficits in the spatial learning ability through enhancing BDNF and TrkB expressions in the ADHD rats. Treadmill exercise for 30 min per day can be considered as the most effective therapeutic modality for the ADHD symptoms.

Keywords: Attention-deficit/hyperactivity disorder, Treadmill exercise, Spatial learning ability, Brain-derived neurotrophic factor, Tyrosine kinase $B$

\section{INTRODUCTION}

Attention-deficit/hyperactivity disorder (ADHD) is a common psychiatric disorder, and this disorder is characterized by following behavioral symptoms: inattention, hyperactivity, and impulsivity. ADHD patients show impairments in academic, social, and occupational functioning, and they exhibit learning difficulty and impulsiveness (Wilens and Dodson, 2004). In animal study, ADHD rats show deficits in the spatial learning ability with reduced brain-derived neurotrophic factor (BDNF) expression (Kim et al., 2011).
BDNF is a member of the nerve growth factor family of neurotrophins, and BDNF is implicated in the survival and differentiation of neurons (Spina et al., 1992). Loss of BDNF during earlier stages of development caused hyperactivity and more pronounced hippocampal-dependent learning deficits (Monteggia et al., 2004). Processing of BDNF was associated with the acquisition of new information and the updating of information about a salient stimulus (Barnes and Thomas, 2008). BDNF works through binding to its high affinity receptor, tyrosine kinase B (TrkB). BDNF-TrkB signaling is involved in synaptic tagging such as long-term potentiation and long-term memory (Lu et al., 2008).
${ }^{*}$ Corresponding author: Seung Wook Choi

Department of Physical Education, Graduate School, Sungshin Women's University, 2 Bomun-ro 34da-gil, Seongbuk-gu, Seoul 136-742, Korea

Tel: +82-2-920-7656, Fax: +82-2-920-7513, E-mail: swchoi@sungshin.ac.kr

Received: June 12, 2014 / Accepted: June 19, 2014
This is an Open Access article distributed under the terms of the Creative Commons Attribution Non-Commercial License (http://creativecommons.org/licenses/by-nc/3.0/) which permits unrestricted non-commercial use, distribution, and reproduction in any medium, provided the original work is properly cited. 
BDNF is associated with the pathophysiology of several psychiatric diseases, including mood disorders (Strauss et al., 2005). Suppressed BDNF level is memory deficits induced by several neuropsychiatric diseases (Hwang et al., 2013; Kim et al., 2011; Sim, 2014).

Exercise is known to improve learning ability and memory function (Jee et al., 2008; Kim et al., 2010). In ADHD children, physical movement improved working speed and social behavioral problems, and diminished hyperactivity (Majorek et al., 2004). Treadmill exercise diminished hyperactivity and improved spatial learning ability in ADHD rats (Kim et al., 2011). Ji et al. (2014) evaluated the duration-dependence of the effect of treadmill exercise on hyperactivity. They concluded that treadmill exercise for 30 min once a day showed most potent inhibitory effect on hyperactivity in ADHD rats.

In the present study, we investigated the duration-dependence of the effect of treadmill exercise on spatial learning ability in relation with BDNF expression in ADHD rats. For this study, radial 8-arm maze test and western blot for BDNF and TrkB were performed.

\section{MATERIALS AND METHODS}

\section{Experimental animals and treatment}

Adult male spontaneous hypertensive rats (SHR) weighing (210 $\pm 10 \mathrm{~g}$ ) were used as the ADHD rats, because the SHR displays the major symptoms of ADHD, such as inattention, hyperactivity, and impulsiveness (Sagvolden, 2000). SHR was selected only when showed hyperactivity in open field test. Meanwhile, Wistar-Kyoto rats (WKYR) weighing $(210 \pm 10 \mathrm{~g})$ were used as the control rats, according to the previous study (Sagvolden, 2000). The rats were housed under controlled temperature $\left(20 \pm 2^{\circ} \mathrm{C}\right)$ and lighting conditions (07:00-19:00), with food and water made available ad libitum throughout the experiments. The animals were randomly divided into four groups $(\mathrm{n}=10$ in each group): control group, ADHD group, ADHD and 10 min treadmill exercise group, ADHD and 30 min treadmill exercise group, and ADHD and 60 min treadmill exercise group. All animal procedures were performed in accordance with the animal care guidelines of the National Institutes of Health (NIH) and the Korean Academy of Medical Sciences.

\section{Treadmill exercise protocol}

The rats in the treadmill exercise groups were subjected to run on a treadmill for $10 \mathrm{~min}, 30 \mathrm{~min}$, and $60 \mathrm{~min}$ once a day, five times a week, for 28 days, according to each group. Exercise load for the running group consisted of running at a speed of $2 \mathrm{~m} / \mathrm{min}$ for the first $5 \mathrm{~min}$, at a speed of $5 \mathrm{~m} / \mathrm{min}$ for the next $5 \mathrm{~min}$, and then at a speed of $8 \mathrm{~m} / \mathrm{min}$ for the last $20 \mathrm{~min}$, with the $0^{\circ}$ inclination.

\section{Radial 8-arm maze test}

Spatial learning ability was determined using a radial 8-arm maze apparatus, as the previously described method (Kim et al., 2006; Sim et al., 2014). The radial 8-arm maze apparatus consisted of a central octagonal plate $(30 \mathrm{~cm}$ in diameter) and radiating eight arms $(50 \mathrm{~cm}$ in length and $10 \mathrm{~cm}$ in width). The apparatus was placed $1 \mathrm{~m}$ above the floor. A small receptacle filled with water $(3 \mathrm{~cm}$ in diameter and $1 \mathrm{~cm}$ in depth) was located at the end of the arms. The rats were trained three times before the spatial learning test. The rats deprived of water for $24 \mathrm{~h}$ were allowed to explore the water and to drink for $5 \mathrm{~min}$. On the 28 days after the starting of experiment, the spatial learning ability was determined. The time spent in seeking water at the end of the arms was counted. The test was terminated when a rat found water in all eight arms or over 6 min elapsed. Re-entering to the previously visited arms was counted as an error. In addition, the number of correct choice before the first error was counted.

\section{Tissue preparation}

The rats were sacrificed, immediately after determining spatial learning ability. To begin the sacrifice process, the animals were fully anesthetized using Zoletil $50^{\circledR}(10 \mathrm{mg} / \mathrm{kg}$, i.p.; Vibac Laboratories, Carros, France). The anesthetized rats were transcardially perfused with $50 \mathrm{mM}$ phosphate-buffered saline (PBS), and fixed with a freshly prepared solution consisting of $4 \%$ paraformaldehyde (PFA) in $100 \mathrm{mM}$ phosphate buffer (PB) at $\mathrm{pH} 7.4$. Brains were dissected, post-fixed in the same fixative overnight, and transferred to $30 \%$ sucrose for cryoprotection. Coronal sections of $40 \mu \mathrm{m}$ thickness were made with a freezing microtome (Leica, Nussloch, Germany).

\section{Western blot analysis for BDNF and TrkB expressions}

BDNF and TrkB expressions were determined by western blot, as the previously described method (Kim et al., 2010; Sim et al., 2014). The hippocampal tissues were collected, and then were immediately frozen at $-70^{\circ} \mathrm{C}$. The hippocampal tissues were homogenized on ice, and lysed in a lysis buffer containing $50 \mathrm{mM}$ HEPES (pH 7.5), $150 \mathrm{mM} \mathrm{NaCl}, 10 \%$ glycerol, $1 \%$ Triton X-100, 1 mM PMSF, 1 mM EGTA, $1.5 \mathrm{mM} \mathrm{MgCl} 2 \cdot 6 \mathrm{H}_{2} \mathrm{O}, 1$ 
$\mathrm{mM}$ sodium orthovanadate, and $100 \mathrm{mM}$ sodium flouride. Protein content was measured using a Bio-Rad colorimetric protein
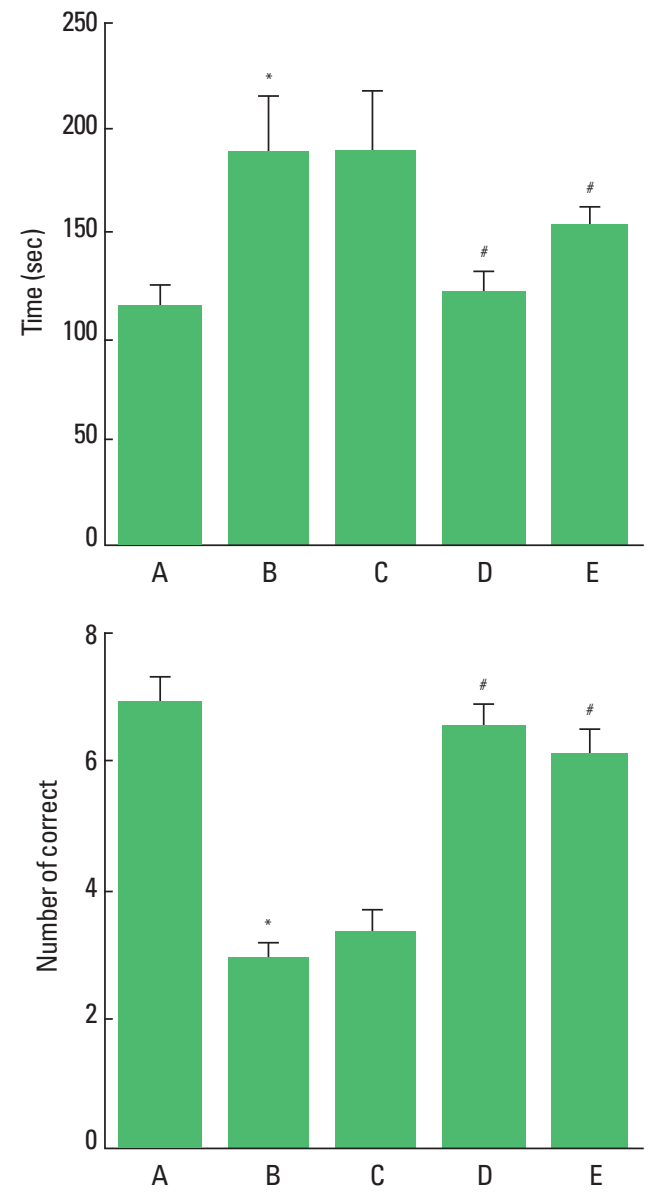

B

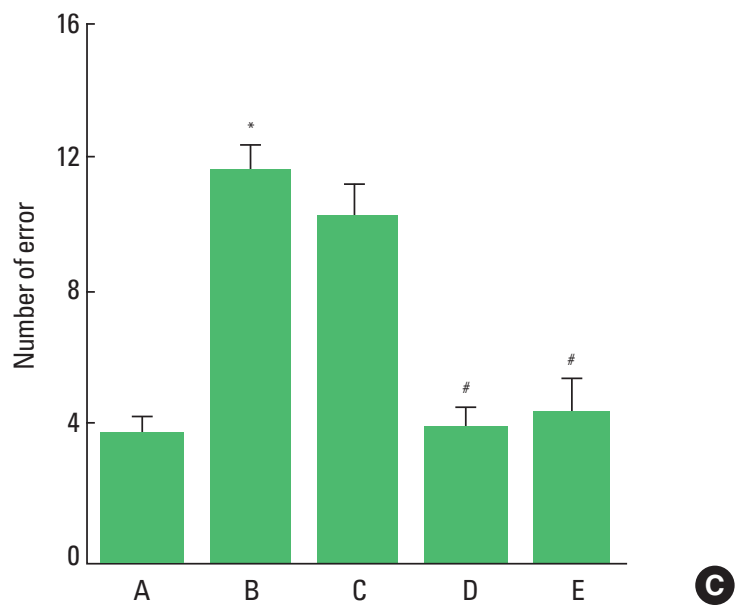

Fig. 1. Effects of treadmill exercise on performance time, correct number, and error number in the radial 8-arm maze test. (A) Control group, (B) ADHD group, (C) ADHD and 10 min treadmill exercise group, (D) ADHD and 30 min treadmill exercise group, and (E) ADHD and 60 min treadmill exercise group. *Represents $P<0.05$ compared to the control group. ${ }^{*}$ Represents $P<0.05$ compared to the $\mathrm{ADHD}$ group. assay kit (Bio-Rad, Hercules, CA, USA). Protein $(30 \mu \mathrm{g})$ was separated on SDS-polyacrylamide gels and transferred onto a nitrocellulose membrane.

Mouse beta-actin antibody (1:500; Santa Cruz Biotech, Santa Cruz, CA, USA), rabbit BDNF antibody (1:1,000; Santa Cruz Biotechnology), rabbit TrkB antibody (1:1,000; Santa Cruz Biotechnology) were used as the primary antibodies. Horseradish peroxidase-conjugated anti-mouse antibody for actin and anti-rabbit antibody for BDNF and TrkB (1:3,000; Vector Laboratories, Burlingame, CA, USA) were used as the secondary antibodies.

Experiment was performed in normal lab conditions and at room temperature except membrane transfer. Membrane transfer was performed at $4^{\circ} \mathrm{C}$ with the cold pack and pre-chilled buffer. Band detection was performed using the enhanced chemiluminescence (ECL) detection kit (Santa Cruz Biotechnology).

\section{Data analysis}

To compare the relative expression of proteins, the detected bands were calculated densitometrically using Molecular Analyst ${ }^{\mathrm{TM}}$, version 1.4.1 (Bio-Rad). Statistical analysis was performed using one-way ANOVA followed by Duncan's post-hoc test, and the results were expressed as the mean \pm standard error of the mean (SEM). Significance was set as $P<0.05$.

\section{RESULTS}

\section{Effects of treadmill exercise on spatial learning ability in the radial 8-arm maze test}

The performance time, number of correct, and number of error choice in the radial 8-arm maze test are presented in Fig. 1. The performance time was $116.60 \pm 7.61 \mathrm{sec}$ in the control group, $189.20 \pm 26.56 \mathrm{sec}$ in the ADHD group, $190.60 \pm 27.44 \mathrm{sec}$ in the ADHD and 10 min treadmill exercise group, $123.40 \pm 8.52 \mathrm{sec}$ in the ADHD and 30 min treadmill exercise group, and $154.80 \pm$ $8.48 \mathrm{sec}$ in the $\mathrm{ADHD}$ and $60 \mathrm{~min}$ treadmill exercise group.

The number of correct choice before the first error was 7.00 \pm 0.36 in the control group, $3.00 \pm 0.21$ in the ADHD group, $3.40 \pm 0.33$ in the ADHD and 10 min treadmill exercise group, $6.60 \pm 0.33$ in the ADHD and 30 min treadmill exercise group, and $6.20 \pm 0.38$ in the ADHD and 60 min treadmill exercise group.

The number of error made before eight successful performances was $3.80 \pm 0.48$ in the control group, $11.60 \pm 0.80$ in the ADHD group, $10.40 \pm 0.85$ in the ADHD and 10 min treadmill exercise group, $4.20 \pm 0.48$ in the ADHD and 30 min treadmill exercise 
group, and $4.60 \pm 0.95$ in the ADHD and 60 min treadmill exercise group.

These results demonstrated that ADHD rats showed longer performance time, lower correct number, and higher error number than those in the control rats. Treadmill exercise shortened performance time, enhanced correct number, and decreased error number. Treadmill exercise for 30 min showed most potent effects on these parameters.

\section{Effects of treadmill exercise on BDNF and TrkB expressions in the hippocampus}

The expressions of BDNF and TrkB in the hippocampus were analyzed by western blotting (Fig. 2). When the level of BDNF $(14 \mathrm{kDa})$ in the control group was set as 1.00 , the level of BDNF was $0.37 \pm 0.03$ in the ADHD group, $0.46 \pm 0.04$ in the $\mathrm{ADHD}$ and 10 min treadmill exercise group, $0.93 \pm 0.02$ in the ADHD and 30 min treadmill exercise group, and $0.94 \pm 0.03$ in the ADHD and 60 min treadmill exercise group.

When the level of mature TrkB (95 kDa) in the control group was set as 1.00, the level of TrkB was $0.20 \pm 0.02$ in the ADHD group, $0.24 \pm 0.02$ in the $\mathrm{ADHD}$ and 10 min treadmill exercise group, $0.44 \pm 0.03$ in the ADHD and 30 min treadmill exercise group, and $0.43 \pm 0.03$ in the ADHD and 60 min treadmill exercise group.

These results showed that expressions of BDNF and TrkB in the hippocampus were decreased in the rats of $\mathrm{ADHD}$ group than the control rats. In contrast, treadmill exercise enhanced expressions of BDNF and TrkB in the ADHD rats. Treadmill exercise for 30 min most potently enhanced the expressions of BDNF and TrkB.

\section{DISCUSSION}

SHR is the most commonly used animal model for ADHD, because SHR strain shows hyperactivity and memory deficits (Sagvolden, 2000). SHR display hyperactivity in the open field test and deficits in the spatial learning ability, while control WKY rats did not show any behavior symptoms of ADHD (Ji et al., 2014; Kim et al., 2011). The beneficial effects of exercise on brain functions are well documented (Ferris et al., 2007; Jee et al., 2008; Sim et al., 2014). Kim et al. (2011) showed that treadmill exercise for $30 \mathrm{~min}$ per day showed most potent alleviating effect on hyperactivity in the ADHD rats. Yun et al. (2014) reported that treadmill exercise ameliorated motor disturbance through reducing Purkinje cell loss and inhibiting astrocyte reaction in the cere- bellum of ADHD rats.

In the present results, ADHD rats displayed impairment of spatial learning ability, in contrast treadmill exercise ameliorated impairment of spatial learning ability in the ADHD rats. Treadmill exercise for 10 min per day did not exert any significant effects,
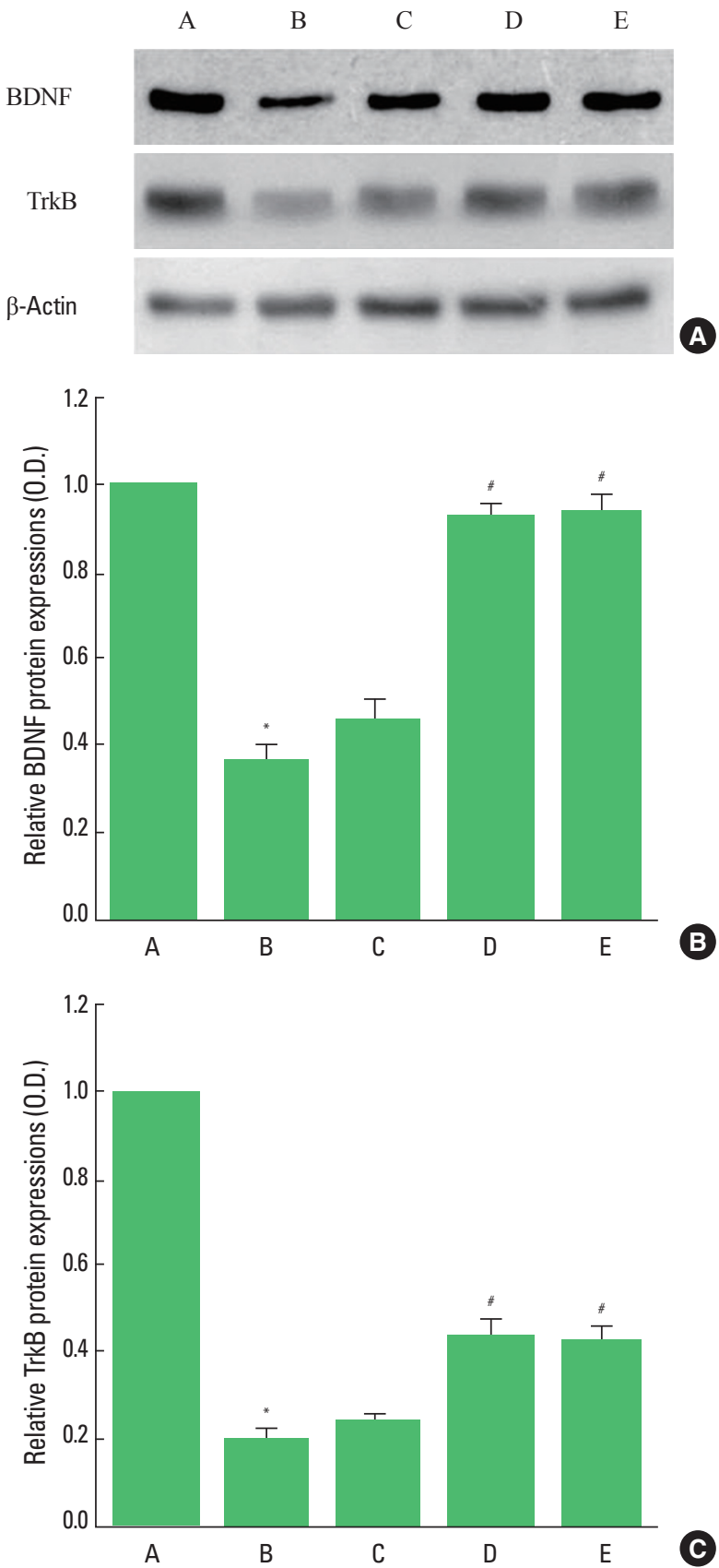

Fig. 2. Effects of treadmill exercise on BDNF and TrkB expressions in the hippocampus. (A) Control group, (B) ADHD group, (C) ADHD and 10 min treadmill exercise group, (D) ADHD and 30 min treadmill exercise group, and (E) ADHD and 60 min treadmill exercise group. ${ }^{*}$ Represents $P<0.05$ compared to the control group. "Represents $P<0.05$ compared to the ADHD group. 
meanwhile treadmill exercise for $30 \mathrm{~min}$ per day showed most potent ameliorating effect on impairment of spatial learning ability.

In BDNF knockout mice, recombinant BDNF completely reversed deficits in long-term potentiation and improved disturbance in synaptic transmission (Patterson et al., 1996). BDNF and TrkB regulate the survival and differentiation of neurons during development, and they are also involved in the several functions in adulthood, including plasticity processes. Alterations of BDNF expression might contribute to the depression, epilepsy, Alzheimer's disease, and Parkinson's disease (Tapia-Arancibia et al., 2004). Increase of plasma BDNF level was observed in the ADHD patients, and plasma BDNF level had a significant positive correlation with the severity of inattention symptoms (Shim et al., 2008). BDNF has pleiotropic effects on neuronal development and synaptic plasticity, and defects in the BDNF functions contribute to cognitive dysfunction and anxiety-like behaviors (Greenberg et al., 2009). BDNF expression in the hippocampus was also suppressed in the old-aged rats with disturbance of short-term memory and spatial learning ability (Kim et al., 2010). There are many reports that treadmill exercise increases BDNF expression in the hippocampus (Kim et al., 2010; Sim et al., 2014; Soya et al., 2007).

In the present results, BDNF and TrkB expressions in the hippocampus were decreased in the ADHD rats, in contrast treadmill exercise enhanced BDNF and TrkB expressions in the ADHD rats. Treadmill exercise for $10 \mathrm{~min}$ per day did not exert any significant effects, meanwhile treadmill exercise for $30 \mathrm{~min}$ and for 60 min per day showed enhancing effects on BDNF and TrkB expressions.

Based on the present study, deficits of spatial learning ability in the ADHD rats can be ascribed to the suppressed BDNF and TrkB expressions. Treadmill exercise alleviated deficits in the spatial learning ability through enhancing BDNF and TrkB expressions in the ADHD rats. Treadmill exercise for 30 min per day can be considered as the most effective therapeutic modality for the ADHD symptoms.

\section{CONFLICT OF INTEREST}

No potential conflict of interest relevant to this article was reported.

\section{REFERENCES}

Barnes P, Thomas KL. Proteolysis of proBDNF is a key regulator in the formation of memory. PLoS One 2008;3:e3248.

Ferris LT, Williams JS, Shen CL. The effect of acute exercise on serum brain-derived neurotrophic factor levels and cognitive function. Med Sci Sports Exerc 2007;39:728-734.

Greenberg ME, Xu B, Lu B, Hempstead BL. New insights in the biology of BDNF synthesis and release: implications in CNS function. J Neurosci 2009;29:12764-12767.

Hwang L, Choi IY, Kim SE, Ko IG, Shin MS, Kim CJ, Kim SH, Jin JJ, Chung JY, Yi JW. Dexmedetomidine ameliorates intracerebral hemorrhage-induced memory impairment by inhibiting apoptosis and enhancing brain-derived neurotrophic factor expression in the rat hippocampus. Int J Mol Med 2013;31:1047-1056.

Jee YS, Ko IG, Sung YH, Lee JW, Kim YS, Kim SE, Kim BK, Seo JH, Shin MS, Lee HH, Cho HJ, Kim CJ. Effects of treadmill exercise on memory and c-Fos expression in the hippocampus of the rats with intracerebroventricular injection of streptozotocin. Neurosci Lett 2008;443:188192.

Ji ES, Kim CJ, Park JH, Bahn GH. Duration-dependence of the effect of treadmill exercise on hyperactivity in attention deficit hyperactivity disorder rats. J Exerc Rehabil 2014;10:75-80.

Kim H, Heo HI, Kim DH, Ko IG, Lee SS, Kim SE, Kim BK, Kim TW, Ji ES, Kim JD, Shin MS, Choi YW, Kim CJ. Treadmill exercise and methylphenidate ameliorate symptoms of attention deficit/hyperactivity disorder through enhancing dopamine synthesis and brain-derived neurotrophic factor expression in spontaneous hypertensive rats. Neurosci Lett 2011;504:35-39.

Kim H, Lee MH, Chang HK, Lee TH, Lee HH, Shin MC, Shin MS, Won R, Shin HS, Kim CJ. Influence of prenatal noise and music on the spatial memory and neurogenesis in the hippocampus of developing rats. Brain Dev 2006;28:109-114.

Kim SE, Ko IG, Kim Bk, Shin MS, Cho S, Kim CJ, Kim SH, Baek SS, Lee EK, Jee YS. Treadmill exercise prevents aging-induced failure of memory through an increase in neurogenesis and suppression of apoptosis in rat hippocampus. Exp Gerontol 2010;45:357-365.

Lu Y, Christian K, Lu B. BDNF: a key regulator for protein synthesis-dependent LTP and long-term memory? Neurobiol Learn Mem 2008; 89:312-323.

Majorek M, Tüchelmann T, Heusser P. Therapeutic Eurythmy-movement therapy for children with attention deficit hyperactivity disorder (ADHD): a pilot study. Complement Ther Nurs Midwifery 2004; 10:46-53.

Monteggia LM, Barrot M, Powell CM, Berton O, Galanis V, Gemelli T, Meuth S, Nagy A, Greene RW, Nestler EJ. Essential role of brain-derived neurotrophic factor in adult hippocampal function. Proc Natl Acad Sci U S A 2004;101:10827-10832. 
Patterson SL, Abel T, Deuel TA, Martin KC, Rose JC, Kandel ER. Recombinant BDNF rescues deficits in basal synaptic transmission and hippocampal LTP in BDNF knockout mice. Neuron 1996;16:1137-1145.

Sagvolden T. Behavioral validation of the spontaneously hypertensive rat (SHR) as an animal model of attention-deficit/hyperactivity disorder (AD/HD). Neurosci Biobehav Rev 2000; 24:31-39.

Shim SH, Hwangbo Y, Kwon YJ, Jeong HY, Lee BH, Lee HJ, Kim YK. Increased levels of plasma brain-derived neurotrophic factor (BDNF) in children with attention deficit-hyperactivity disorder (ADHD). Prog Neuropsychopharmacol Biol Psychiatry 2008;32:1824-1828.

Sim YJ. Treadmill exercise alleviates impairment of spatial learning ability through enhancing cell proliferation in the streptozotocin-induced Alzheimer's disease rats. J Exerc Rehabil 2014;10:81-88.

Soya H, Nakamura T, Deocaris CC, Kimpara A, Iimura M, Fujikawa T, Chang H, McEwen BS, Nishijima T. BDNF induction with mild exercise in the rat hippocampus. Biochem Biophys Res Commun 2007;358: 961-967.

Spina MB, Squinto SP, Miller J, Lindsay RM, Hyman C. Brain derived neurotrophic factor protects dopamine neurons against 6-hydroxydopamine and N-methyl-4-phenylpyridinium ion toxicity: involvement of the glutathione system. J Neurochem 1992;59:99-106.

Strauss J, Barr CL, George CJ, Devlin B, Vetró A, Kiss E, Baji I, King N, Shaikh S, Lanktree M, Kovacs M, Kennedy JL. Brain-derived neurotrophic factor variants are associated with childhood-onset mood disorder: confirmation in a Hungarian sample. Mol Psychiatry 2005;10: 861-867.

Tapia-Arancibia L, Rage F, Givalois L, Arancibia S. Physiology of BDNF: focus on hypothalamic function. Front Neuroendocrinol 2004;25:77107.

Wilens TE, Dodson W. A clinical perspective of attention-deficit/hyperactivity disorder into adulthood. J Clin Psychiatry 2004;65:1301-1313.

Yun HS, Park MS, Ji ES, Kim TW, Ko IG, Kim HB, Kim H. Treadmill exercise ameliorates symptoms of attention deficit/hyperactivity disorder through reducing Purkinje cell loss and astrocytic reaction in spontaneous hypertensive rats. J Exerc Rehabil 2014;10:22-30. 